\title{
Legislación e instrumentos de manejo existentes para la protección de los fondos marinos en la Plataforma Continental Argentina
}

\author{
EsTEBAN GAITÁN
}

Instituto Nacional de Investigación y Desarrollo Pesquero (INIDEP), Paseo Victoria Ocampo No 1, Escollera Norte, B7602HSA - Mar del Plata, Argentina

Marine and

Fishery Sciences

MAFIS

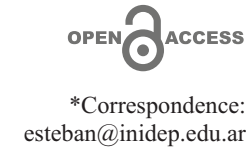

Received: 25 May 2020 Accepted: 29 July 2020

ISSN 2683-7595 (print) ISSN 2683-7951 (online)

https://ojs.inidep.edu.ar

Journal of the Instituto Nacional de Investigación y Desarrollo Pesquero (INIDEP)

This work is licensed under Creative Commons AttributionNonCommercial-ShareAlike 4.0 International License
RESUMEN. Se presenta un compendio de instrumentos vinculantes y no vinculantes referidos al manejo y protección de los fondos marinos y/o de los invertebrados bentónicos de la Plataforma Continental Argentina (PCA). Se analizaron leyes nacionales y acuerdos internacionales en los cuales la Argentina es signataria, así como declaraciones de FAO que establecen principios y normas aplicables a la conservación, ordenación y desarrollo de las pesquerías y ecosistemas asociados. Sobre la base de esta selección bibliográfica se analizó la evolución de las medidas a lo largo del tiempo en función del contexto internacional, a fin de esclarecer cuáles son los faltantes y los logros obtenidos en este proceso. Se concluye que la Argentina avanzó en la generación de políticas acordes a los lineamientos mundiales que implican un manejo ecosistémico y un mayor resguardo de la biodiversidad, por ejemplo, implementando Áreas Marinas Protegidas, así como con el enunciado de planes de investigación y fomento que involucran el estudio de los fondos marinos. Sin embargo, para algunas medidas su efectiva ejecución es parcial a partir de la falta de reglamentación y/o financiamiento. Asimismo, queda pendiente la elaboración de planes de acción concretos referidos al estudio del lecho marino incluido en los nuevos límites exteriores de la PCA.

Palabras clave: Invertebrados bentónicos, conservación, Áreas Marinas Protegidas, enfoque ecosistémico.

Existing legislation and management instruments for the protection of seabed in the Argentine Continental Shelf

ABSTRACT. A compendium of binding instruments and non-binding instruments referring to the management and protection of seabed and/or benthic invertebrates of the Argentine Continental Shelf (ACS) is presented. National laws and international agreements to which Argentina is a signatory were analyzed, as well as FAO declarations that established principles and norms applicable to the conservation, management and development of fisheries and associated ecosystems. Based on this selected bibliography, the evolution of measures over time was analyzed according to the international context, in order to clarify what are the shortcomings and the achievements obtained in this process. It is concluded that Argentina advanced in the generation of policies in accordance with global guidelines that imply an ecosystem management and a greater protection of biodiversity, for example implementing Marine Protected Areas, as well as the statement of research and promotion plans that involve the study of the seabed. However, effective execution of some measures is partially accomplished due to the lack of regulation and/or financing. Preparation of research plans related to the study of the seabed included in the new outer limits of the ACS is pending.

Key words: Benthic invertebrates, conservation, Marine Protected Areas, ecosystem approach. 


\section{INTRODUCCIÓN}

Existen numerosas medidas de ordenación que buscan minimizar los impactos de las actividades extractivas a gran escala, de forma tal que se pueda mantener una explotación sostenible de las mismas. En el caso de los recursos acuáticos y la pesca en particular, en los últimos años estas medidas han prestado atención no solo al estado de los recursos pesqueros sino también al entorno donde estos habitan. Por ejemplo, los fondos marinos pueden ser fuertemente afectados por las actividades pesqueras, y para mitigar estos efectos se ha construido un cuerpo de instrumentos vinculantes (leyes, tratados, resoluciones) y no vinculantes (declaraciones de principios o códigos de FAO) que en distinto grado hacen mención a la necesidad de proteger estos ambientes de las acciones generadas por el hombre.

Estas iniciativas nacieron a partir de un cambio de paradigma, cuando se planteó una nueva idea de prácticas pesqueras dejando atrás el manejo mono-específico y poniendo el foco en un manejo integral de las pesquerías. Esto incluyó a las comunidades de invertebrados bentónicos constituidas por aquellos organismos que viven en relación con el fondo, ya sea por desplazarse sobre él, nadar en su cercanía sin alejarse, o por vivir fijos o enterrados en el lecho marino. Esta localización, asociada al hábitat de distintos recursos demersales y/o bentónicos, hace que estos organismos sean vulnerables a las pesquerías que operan con redes de arrastre de fondo. De hecho, relacionado con este cambio de paradigma, se comprobaron numerosas evidencias del efecto negativo generado por las pesquerías de arrastre de fondo sobre los invertebrados bentónicos y su ambiente (Collie et al. 2000; Johnson 2002; Thrush y Dayton 2002). Estos efectos incluyen la pérdida de biodiversidad (Cryer et al. 2002; Cook et al. 2013), los cambios en la composición de las comunidades (Tillin et al. 2006), la homogeneiza- ción del ambiente (Thrush et al. 2006) e incluso la disminución en el reclutamiento de peces (Auster et al. 1996; Muntadas et al. 2014).

En ese marco surgieron las primeras directrices internacionales a través de FAO, en donde se comenzó a considerar la pesca desde un punto de vista holístico. En 1995 FAO presentó el Código de Conducta para la Pesca Responsable que sugiere que "las medidas de ordenación deberían asegurar la conservación no solo de las especies objetivo, sino también de aquellas especies pertenecientes al mismo ecosistema o dependientes de ellas o que están asociadas con ellas". Posteriormente, FAO publicó en 2003 el documento técnico "Enfoque ecosistémico para las pesquerías", en donde define este enfoque como "...una extensión del manejo pesquero convencional que reconoce más explícitamente la interdependencia entre el bienestar humano y la salud de los ecosistemas y la necesidad de mantener su productividad para las generaciones presentes y futuras".

En el caso particular de la Argentina, los primeros indicios sobre una planificación gubernamental de investigaciones marinas que involucran los fondos marinos se esbozan en 1988. En ese año el Comité Científico-Técnico del Mar Argentino (CO.CI.TE.MA.) elaboró un Plan Oceanográfico Argentino para el quinquenio 1990-1995. Sobre esta base se desarrolló el Programa Oceanográfico Argentino que mencionaba que “...el conocimiento del Mar Epicontinental Argentino y el de su Plataforma Submarina debe ser sensiblemente incrementado". Seguidamente, en 1996 se creó la Comisión de Estudios del Mar Argentino, la cual propuso un capítulo específico sobre el Mar Argentino en el Plan Nacional Plurianual de Ciencia y Tecnología 1999-2001. Uno de los objetivos de este plan era la idea de concentrar los esfuerzos de investigación en tres áreas: recursos vivos, recursos no vivos y medio ambiente. Cabe destacar que más allá de la mención indirecta de los fondos marinos ninguna de estas iniciativas finalmente tuvo un desarrollo efectivo (González 2012). 
Más cerca en el tiempo, un hecho concreto referido a un manejo integral de la pesca en nuestro país fue la declaración del Consejo Federal Pesquero $N^{\circ} 26$ de 2006. Esta instruye al Instituto Nacional de Investigación y Desarrollo Pesquero (INIDEP) para "la puesta en funcionamiento de programas de investigación... estructurados bajo un enfoque ecosistémico...". Otro hito importante fue la creación en 2013 de la primera Área Marina Protegida (AMP) oceánica NamuncuráBanco Burdwood cuyos objetivos son "conservar una zona de alta sensibilidad ambiental y de importancia para la protección y gestión sostenible de la biodiversidad de los fondos marinos" y "promover el manejo sostenible, ambiental y económico de los ecosistemas marinos bentónicos de nuestra plataforma..."

Como se verá más adelante, estas apreciaciones cobran mayor sentido en el marco de los nuevos límites exteriores de la Plataforma Continental Argentina (PCA). Estos fueron redefinidos luego de la presentación de la Comisión Nacional del Límite Exterior de la Plataforma Continental (COPLA) y de la recomendación de la Comisión de Límites de la Plataforma Continental (creada por la CONVEMAR y con sede en la ONU). A través de este nuevo límite exterior de la plataforma, la Argentina adquiere la soberanía sobre el lecho, subsuelo y los recursos vivos bentónicos en un sector que abarca $1.785 .000 \mathrm{~km}^{2}$ (COPLA 2017). De esta manera, nace la oportunidad para un enorme campo de investigación sobre la presencia de invertebrados bentónicos que sean pasibles de ser sometidos a una explotación sostenible o que presenten una alta relevancia ecológica por su vulnerabilidad, tales como los corales y esponjas formadores de "bosques animales" (Rossi et al. 2017), los cuales tienen amplio desarrollo en otros sectores de la PCA (Schejter et al. 2020). Del mismo modo, la posibilidad de exploraciones de recursos hidrocarburíferos en este sector necesita ser acompañada de un relevamiento que establezca una línea de base a fin de realizar futuras evaluaciones de impacto ambiental.
Si bien existen distintos trabajos donde se ha revisado el estado y la evolución de las medidas tendientes a la ordenación del medio costero y marino en aguas argentinas, estos están principalmente enfocados en las Áreas Marinas Protegidas (AMP's) y sus aspectos jurídicos asociados (FAO 2012; Radovich 2018; Cuminetti 2020). Sin embargo, a la fecha no existen trabajos específicos que aborden el tema desde el punto de vista del manejo y protección de los fondos marinos y/o de las especies de invertebrados bentónicos que se desarrollan en dicho ambiente. Por lo antedicho, en este trabajo se presenta un compendio de las distintas instancias legales e institucionales donde se hace mención a dicho ambiente y se analiza su evolución a lo largo del tiempo en función del contexto internacional, a fin de esclarecer cuales son los faltantes y los logros obtenidos en este proceso. Esto servirá de guía para la implementación de planes de investigación referidos a los fondos marinos con las justificaciones pertinentes.

\section{METODOLOGÍA Y FUENTES DE INFORMACIÓN}

Se compiló la información referida a la conservación, gestión e investigación de los fondos marinos que se encuentra presente en la legislación nacional (leyes) e internacional (tratados donde la Argentina es signataria). No se incluyen en este ítem aquellas medidas que involucran parques marinos provinciales o inter-jurisdiccionales (ver Cuminetti 2020 para detalles específicos de esto). Para cada uno de los elementos analizados y con base en su interpretación, se discriminó si las referencias a los fondos marinos y/o bentos eran de forma directa o indirecta y se transcribieron sus principales aspectos y/o recomendaciones. También se examinaron distintos planes nacionales de investigación y fomento planteados en la Argentina a partir de 2010 en los cuales se 
establece el estudio de las comunidades bentónicas como uno de los ítems relevantes a ser desarrollados. Asimismo, se analizaron las declaraciones formuladas por FAO que, de manera no obligatoria, establecen principios y normas aplicables a la conservación, ordenación y desarrollo de las pesquerías y ecosistemas asociados.

\section{RESULTADOS}

\section{Legislación a nivel nacional}

Todas las normas existentes son posteriores a 1990, iniciando con la aprobación de la Ley de Espacios Marítimos No 23.968 en 1991 y el establecimiento allí de los límites de la PCA como primera referencia sobre los fondos marinos. Esto ocurrió dado que la Argentina adhirió a la Convención de las Naciones Unidas sobre el Derecho del Mar (CONVEMAR) recién en 1995 a partir de la sanción de la Ley No 24.543 (Tabla 1).

Seguidamente, en la reforma de la Constitución Nacional de 1994, se produjo la inclusión del artículo 41 denominado cláusula ambiental en donde se hace una primera mención a la explotación sostenible del ambiente. Se sumó luego la Ley Federal de Pesca No 24.922 en 1997, que establece a la Secretaría de Agricultura, Ganadería, Pesca y Alimentos (SAGPyA) como órgano de aplicación de la ley y crea el Consejo Federal Pesquero (CFP). Éste último regula la actividad pesquera en función de medidas basadas en la conservación de los recursos, la pesca sostenible y la protección del ecosistema a fin de evitar la sobreexplotación y prevenir los efectos negativos sobre el ambiente y los sistemas ecológicos. Un ejemplo de esto es la aplicación de zonas de veda designadas por el CFP donde se prohíben los arrastres de fondo, medida que si bien está dirigida a la protección de especies de interés pesquero puede redundar en un efecto positivo sobre la comunidad bentónica.
Otra medida fue la aprobación de la Ley General del Ambiente $N^{\circ} 25.675$ en 2002, la cual establece los presupuestos mínimos para el logro de una gestión sostenible del ambiente, con foco en la preservación y protección de la diversidad biológica. Esta ley apela al "principio precautorio", estableciendo que la falta de información no es una razón para posponer medidas de conservación e instaura la necesidad de evaluaciones de impacto ambiental previo al desarrollo de iniciativas que puedan afectar al ambiente. Por último, y a partir de la Iniciativa Pampa Azul (que se detalla más adelante) surgieron distintas leyes relacionadas con la creación de Áreas Marinas Protegidas (AMP's) como la Ley No 26.875: Área Marina Protegida Namuncurá-Banco Burdwood y la Ley $\mathrm{N}^{\mathrm{o}}$ 27.037: Sistema Nacional de Áreas Marinas Protegidas, así como otras relacionadas con el financiamiento necesario para realizar las investigaciones en dichos sectores (Ley $N^{o}$ 27.167: Programa Nacional de Investigación e Innovación Productiva en Espacios Marítimos Argentinos -PROMAR).

Hay que resaltar que en el contexto del Sistema Nacional de Áreas Marinas Protegidas, a fines de 2018 se aprobó por el Congreso Nacional (Ley N ${ }^{\circ}$ 27.490) la creación de dos nuevas AMP's: Banco Burdwood II y Yaganes. En el caso del AMP Banco Burdwood II, uno de los fundamentos para su creación fue la presencia de organismos bentónicos estructurantes de "bosques animales", generando una zona de gran diversidad, alto nivel de endemismo y lenta recuperación en caso de daño. Para el AMP Yaganes se destacan como valores de conservación del área la presencia de taxones indicadores de Ecosistemas Marinos Vulnerables, así como una alta diversidad de especies bentónicas asociadas a corales y vieira patagónica (DNC/APN, 2017). Ambas AMP's junto con la AMP Yaganes-Banco Burdwood preexistente forman hoy parte del Sistema Nacional de Áreas Marinas Protegidas a partir de la adecuación de esta última por el Decreto $\mathrm{N}^{\circ} 888 / 2019$. 
Tabla 1. Aspectos más importantes de la legislación argentina relevantes para la conservación del ambiente bentónico. Table 1. Most important aspects of Argentine legislation relevant to the conservation of the benthic environment.

$\begin{array}{cc}\text { Instrumento legal } & \begin{array}{c}\text { Referencia a fondos marinos } \\ \text { y/o bentos }\end{array}\end{array}$

Ley sobre Espacios

Marítimos $\mathrm{N}^{\mathrm{o}} 23.968$

(1991)

Constitución Nacional

(1994)

Ley Federal de Pesca $\mathrm{N}^{\circ} 24.922$ (1997)

Ley General del Ambiente $\mathrm{N}^{\mathrm{o}} 25.675$ (2002)

Ley Creación del Área Marina Protegida

Namuncurá-Banco

Burdwood No 26.875

(2013)
Directa. Determinación de Plataforma Continental Argentina

Indirecta. Sustentabilidad de actividades productivas

Indirecta. Conservación de los recursos vivos marinos y prevención de daños sobre el entorno. Zonas de veda que limitan arrastres de fondo

Indirecta. Sustentabilidad de actividades productivas. Preservación de diversidad biológica. Evaluaciones de impacto ambiental previas a desarrollo de actividades

Directa. Protección y gestión de ecosistemas marinos bentónicos
Art. 6: "La plataforma continental sobre la cual ejerce soberanía la Nación Argentina, comprende el lecho y el subsuelo de las áreas submarinas que se extienden más allá de su mar territorial y a todo lo largo de la prolongación natural de su territorio hasta el borde exterior del margen continental, o bien hasta una distancia de DOSCIENTAS (200) millas marinas medidas a partir de las líneas de base... en los casos en que el borde exterior no llegue a esa distancia.".

Art. 41 (cláusula ambiental): "Todos los habitantes tienen derecho a gozar de un ambiente sano donde las actividades productivas satisfagan las necesidades presentes sin comprometer las de las generaciones futuras...".

Art. 11: “...correspondiendo al Instituto Nacional de Investigación y Desarrollo Pesquero-INIDEP-, la planificación y ejecución de sus actividades científicas... en lo que se refiere a la evaluación y conservación de los recursos vivos marinos.".

Art. 17: "La pesca en todos los espacios marítimos bajo jurisdicción argentina, estará sujeta a las restricciones que establezca el Consejo Federal Pesquero con fundamento en la conservación de los recursos, con el objeto de evitar excesos de explotación y prevenir efectos dañosos sobre el entorno y la unidad del sistema ecológico.".

Art. 19: “...la Autoridad de Aplicación podrá establecer zonas o épocas de veda...".

Art. 1: "La presente ley establece los presupuestos mínimos para el logro de una gestión sustentable y adecuada del ambiente, la preservación y protección de la diversidad biológica y la implementación del desarrollo sustentable.".

Art. 11: "Toda obra o actividad que... sea susceptible de degradar el ambiente, alguno de sus componentes, o afectar la calidad de vida de la población, en forma significativa, estará sujeta a un procedimiento de evaluación de impacto ambiental, previo a su ejecución.”.

Art. 3: "Son objetivos de la presente ley: a) Conservar una zona de alta sensibilidad ambiental y de importancia para la protección y gestión sostenible de la biodiversidad de los fondos marinos; b) Promover el manejo sostenible, ambiental y económico de los ecosistemas marinos bentónicos de nuestra plataforma a través de un área demostrativa.". 
Tabla 1. Continuación.

Table 1. Continued.

\begin{tabular}{cc}
\hline Instrumento legal & $\begin{array}{c}\text { Referencia a fondos marinos } \\
\mathrm{y} / \mathrm{o} \text { bentos }\end{array}$ \\
\hline
\end{tabular}

Ley Sistema Nacional de Áreas Marinas Protegidas No 27.037 (2014)

Ley Creación Programa Nacional de Investigación e Innovación Productiva en Espacios Marítimos Argentinos (PROMAR) $\mathrm{N}^{\mathrm{o}} 27.167$ (2015)

Ley Creación de dos Áreas Marinas Protegidas (Banco Burdwood II y Yaganes) $N^{\circ} 27.490$ (2018)
Directa. Conservación de ecosistemas bentónicos representativos

Indirecta. Conservación, investigación, uso sustentable de recursos marinos y articulación con iniciativas internacionales para el cumplimiento de lo anterior

Directa. Protección de la biodiversidad bentónica y de especies de interés comercial
Art. 1: "Instituyese...el Sistema Nacional de Áreas Marinas Protegidas, destinado a proteger y conservar espacios marinos representativos de hábitats y ecosistemas...".

Art 2: “...se consideran áreas marinas protegidas a los espacios naturales establecidos para la protección de ecosistemas, comunidades o elementos biológicos o geológicos del medio marino, incluyendo al subsuelo y fondos..."

Art 4: "Las áreas marinas protegidas deberán ser manejadas y utilizadas de una manera sustentable...".

Art. 2: “...objetivos generales: ...e) Implementar planes interdisciplinarios que incluyan la investigación de base, la conservación de las especies y los ambientes marinos... h) Generar insumos científicos para la adopción de políticas de conservación y uso sustentable de los recursos biológicos. j) Articular los programas nacionales con iniciativas internacionales en materia de investigación y conservación marinas...”.

En el caso del AMP Banco Burdwood II uno de los fundamentos es la "presencia en el área de bosques animales, representados por los corales verdaderos (Hexacorallia), como los corales blandos (Octocorallia) y los falsos corales...que generan estructuras que aumentan la diversidad de las comunidades a lo largo del tiempo e identificados como Ecosistemas Marinos Vulnerables". También se señala que es un "Área de alta biodiversidad bentónica y con endemismos para especies de invertebrados con alta fragilidad y de lenta recuperación ante amenazas.".

Para el AMP Yaganes, pueden destacarse entre sus valores de conservación la "Presencia de cañones submarinos sobre el talud Austral y Montes submarinos importantes para la biodiversidad bentónica"; que es un "Área de alta biodiversidad para especies de comunidades bentónicas de relevancia consideradas taxones indicadores de Ecosistemas Marinos Vulnerables" y la "Presencia de crustáceos de interés comercial como la Centolla, Centollón...". 


\section{Legislación a nivel internacional}

La República Argentina ratificó por leyes del Congreso Nacional diversos tratados internacionales que, en todo o en parte, se relacionan con la conservación y el uso sostenible de los recursos marinos bentónicos (Tabla 2).

A nivel regional es relevante citar el Tratado del Río de la Plata y su Frente Marítimo firmado por la Argentina y el Uruguay en 1973 (aprobado por Ley $N^{o} 20.645$ ), dentro del cual se constituye la Comisión Técnica Mixta (CTM). La CTM es la encargada de adoptar medidas concernientes a la conservación, preservación y explotación de recursos acuáticos pero también a la protección del medio marino.

En 1994, a partir de la Ley $\mathrm{N}^{\circ} 24.375$, la Argentina ratifica el Convenio sobre la Diversidad Biológica impulsado por el Programa de las Naciones Unidas para el Medio Ambiente (PNUMA). Este convenio presenta como objetivo principal la conservación de la diversidad biológica y el uso sostenible de sus componentes. Así, los Estados parte se comprometen a monitorear y planificar la protección de la biodiversidad a través de la inclusión de políticas activas de conservación (por ejemplo a partir de la creación de Áreas Protegidas o la implementación de Planes de Acción Nacionales) tratando de reducir al mínimo los efectos adversos de las actividades humanas.

Como se mencionó anteriormente, recién en 1995 la Argentina aprobó la CONVEMAR por medio de la Ley No 24.543: Protección y Preservación del Medio Marino. Allí se estableció la definición de plataforma continental y los derechos del Estado ribereño sobre ella, lo que incluye derechos de soberanía a los efectos de la exploración y de la explotación de sus recursos naturales, tanto los organismos vivos pertenecientes a especies sedentarias como los recursos no vivos del lecho del mar y su subsuelo (minerales, petróleo, etc.).

También como parte de los países firmante del Tratado Antártico nuestro país suscribió en 2000 a la Convención sobre la Conservación de los Recursos Vivos Marinos Antárticos (CCRVMA) mediante la Ley $N^{\circ}$ 25.263: Régimen de recolección de recursos vivos marinos en el área de aplicación de la Convención para la Conservación de los Recursos Vivos Marinos Antárticos (CCRVMA). La CCRVMA incluye dentro de su área de influencia los archipiélagos de las islas Georgias del Sur, Sandwich del Sur y Orcadas del Sur reivindicados como propios por la Argentina y por lo tanto incluidos en la presentación efectuada por la COPLA. Sin perjuicio de esto, la CCRVMA propicia la concreción de AMP's en su ámbito (CCRVMA 2009a) así como la detección y resguardo de Ecosistemas Marinos Vulnerables (CCRVMA 2009b) en función de proteger ambientes bentónicos prioritarios del impacto ejercido por la pesca de fondo.

\section{Planes de investigación y fomento sobre los fondos marinos en la Argentina}

En este apartado se mencionan los principales planes de investigación y fomento diseñados a partir de 2010 referidos a las actividades científico-productivas que incluyen en algún tópico un desarrollo asociado a los fondos marinos y se resumen los ítems sustanciales de cada plan.

Informe para el Plan Estratégico Territorial 2011. Ministerio de Planificación Federal, Inversión Pública y Servicios de la Nación

Este informe brinda los lineamientos para la incorporación de la problemática del Mar Argentino en la planificación territorial, desde una revisión de los distintos aspectos productivos que allí se desarrollan con una visión geopolítica. Hace hincapié en el gran potencial hidrocarburífero del sector adyacente al anterior límite de la plataforma, es decir los fondos marinos y oceánicos fuera de la jurisdicción argentina (llamados "La zona"). Lo más relevante es que allí se muestran las tendencias exploratorias en la búsqueda de hidrocarburos para los próximos años, las cuales 
Tabla 2. Tratados internacionales y sus principales aspectos relacionados con la conservación y el uso sostenible de los recursos marinos bentónicos.

Table 2. International treaties and their main aspects related to the conservation and sustainable use of benthic marine resources.

\begin{tabular}{lc}
\hline $\begin{array}{l}\text { Instrumento legal (ley de } \\
\text { ratificación) }\end{array}$ & Referencia a fondos marinos \\
$y / o$ bentos & Principales aspectos y/o recomendaciones
\end{tabular}

Tratado del Río de la Plata y su Frente Marítimo (Ley No $20.645 / 1973$ )

Convenio Diversidad Biológica (Ley $N^{\circ}$ 24.375/1994)

Convención de las Naciones Unidas sobre el Derecho del Mar (CONVEMAR) (Ley No 24.543/1995)

Comisión para la Conservación de los Recursos Vivos Marinos Antárticos (CCRVMA) (Ley No 25.263/2000)
Directa. Exploración, explotación y conservación de recursos bentónicos

Indirecta. Conservación y uso sostenible de biodiversidad, creación de AMPs, estudios de impacto ambiental

Directa. Exploración y explotación de recursos vivos y no vivos de la plataforma

Directa. Conservación de bio-regiones bentónicas
Art. 41: “Cada parte podrá explorar y explotar los recursos del lecho y del subsuelo del Río en las zonas adyacentes a sus respectivas costas...".

Art. 80: "Las Partes, constituyen una Comisión Técnica Mixta... que tendrá por cometido la realización de estudios y la adopción y coordinación de planes y medidas relativas a la conservación, preservación y racional explotación de los recursos vivos y a la protección del medio marino en la zona de interés común...".

Art. 6: "Cada Parte Contratante... b) Integrará...la conservación y la utilización sostenible de la diversidad biológica en los planes, programas y políticas...".

Art. 8: "Cada Parte Contratante... a) Establecerá un sistema de áreas protegidas...".

Art. 14: "Cada Parte Contratante... a) Establecerá procedimientos apropiados por los que se exija la evaluación del impacto ambiental de sus proyectos propuestos que puedan tener efectos adversos importantes para la diversidad biológica con miras a evitar o reducir al mínimo esos efectos...".

Art. 77: “...1. El Estado ribereño ejerce derechos de soberanía sobre la plataforma continental a los efectos de su exploración y de la explotación de sus recursos naturales. 4. Los recursos naturales... son los recursos minerales y otros recursos no vivos del lecho del mar y su subsuelo, así como los organismos vivos pertenecientes a especies sedentarias...".

Medida de Conservación 22/06 (Protección de la plataforma sur de las Islas Orcadas del Sur): "Consciente de la necesidad de otorgar protección adicional a esta importante región a fin de proporcionar un área de referencia científica, y de conservar ejemplos representativos de bio-regiones bentónicas...”. El área... se designará como área marina protegida, a los efectos de contribuir a la conservación de la biodiversidad marina... Se prohíbe todo tipo de actividad de pesca dentro del área. 
coinciden en gran parte con el sector que a partir de la ampliación de la plataforma continental queda sujeto a jurisdicción nacional. Esto implica un enorme sector con posibilidades de ser explotado desde el punto de vista energético, pero que amerita estudios de base previos sobre las comunidades bentónicas afincadas en esos fondos que puedan ser afectadas por estas explotaciones.

Plan Argentina Innovadora 2020. Plan operativo 2013-2016. Núcleo socio-productivo estratégico: producción y procesamiento de recursos oceánicos. Ministerio de Ciencia, Tecnología e Innovación Productiva

El Plan Argentina Innovadora 2020 tiene como fin fortalecer la base científica y tecnológica con el agregado de valor en intervenciones focalizadas en la resolución de núcleos socio-productivos estratégicos (NSPE), caracterizados por su alto impacto económico, tecnológico o social. En ese contexto, se plantea el núcleo de Producción y procesamiento de recursos oceánicos, el cual incluso es uno de los Temas estratégicos para convocatorias de Ingresos a la Carrera de Investigador Científico en el sector Agroindustria del Consejo Nacional de Investigaciones Científicas y Técnicas (CONICET). Este plan presenta una serie de objetivos, metas y líneas de fomento a la investigación vinculadas con los fondos marinos, ya sea a través de la explotación hidrocarburífera como de la investigación centrada en ecosistemas bentónicos. Asimismo, plantea la necesidad de financiar proyectos de investigación y desarrollo que aumenten el conocimiento de la denominada Economía Azul (Blue Economy), concepto que implica la utilización del mar y sus recursos de forma que se produzca un desarrollo económico sostenible.

Iniciativa Pampa Azul. Ministerio de Ciencia, Tecnología e Innovación Productiva

La iniciativa Pampa Azul fue presentada en 2014 y su objetivo incluye profundizar las políti- cas de conservación, promover innovaciones tecnológicas para explotaciones sostenibles de recursos marinos y concientizar y promover el ejercicio soberano a través de la ciencia en el Atlántico Sur. En la génesis de este proyecto se distinguen cinco zonas prioritarias. En cuatro de ellas (Banco Burdwood, Agujero Azul, Golfo San Jorge e islas subantárticas) las comunidades bentónicas presentan características de particular interés. En algunos casos esto se debe a su alta biodiversidad, fragilidad o endemismo, que las destaca como factores relevantes para la determinación de esas áreas de investigación. En otros casos, algunas de las especies bentónicas presentes en estos sectores (vieira, langostino, centolla) están sometidas a explotación pesquera, por lo que un manejo sostenible de estos recursos requiere de una continua generación de información (Pampa Azul, 2017).

Estrategia nacional sobre la biodiversidad. Plan de acción 2016-2020. Ministerio de Ambiente y Desarrollo Sostenible de la Nación

Este documento sintetiza los compromisos asumidos por la Argentina en el marco del Convenio sobre la Diversidad Biológica $(\mathrm{CDB})$ y la Agenda 2030 de Objetivos de Desarrollo Sostenible de las Naciones Unidas. El marco referencial es el Plan Estratégico de Biodiversidad 20112020 y las Metas de Aichi, con un objetivo general de conservar la biodiversidad con un enfoque basado en los ecosistemas. Si bien tiene un marco amplio, el punto 4 apunta a prácticas productivas y de consumo sostenibles, donde se incluye a la pesca. En el sentido de practicar una pesca sostenible, el documento señala que es necesario balancear el desarrollo productivo con un uso sostenible de la biodiversidad y advierte del fuerte impacto que tienen las redes de arrastre sobre el hábitat bentónico. En ese contexto se planifican las metas nacionales prioritarias, en las cuales la $\mathrm{N}^{\mathrm{o}} 3$ propone alcanzar un $10 \%$ de superficies resguardadas de zonas marinas y costeras de los espacios marítimos argentinos. 


\section{Recomendaciones y resoluciones no vinculan- tes de organismos internacionales}

La Organización de las Naciones Unidas (ONU) a través de FAO dictó distintas recomendaciones relacionadas con la explotación sostenible de los recursos naturales marinos, las cuales sirven de referencia para distintas medidas de ordenación que se han establecido. Dentro de estos instrumentos internacionales no vinculantes, la Argentina aplica el Código de Conducta para la Pesca Responsable y el Enfoque Ecosistémico para la Pesca, mencionados anteriormente y con ejes de acción bastante amplios. En línea con esto, se ha elaborado el Plan de Acción Nacional para Prevenir, Desalentar y Eliminar la Pesca Ilegal, No Declarada y No Reglamentada (PANINDNR) y los planes de acción nacionales (PAN) para aves, mamíferos y tiburones.

La Agenda 2030 de Objetivos de Desarrollo Sostenible aprobada en septiembre de 2015 por la Asamblea General de las Naciones Unidas, también se refiere a la conservación del medio marino. Dentro de sus objetivos de desarrollo sostenible (ODS), el No 14 "conservar y utilizar en forma sostenible los océanos, los mares y los recursos marinos para el desarrollo sostenible", plantea para 2020 gestionar y proteger sosteniblemente los ecosistemas marinos y costeros para evitar efectos adversos importantes, poner fin a las prácticas pesqueras destructivas y conservar al menos el $10 \%$ de las zonas costeras y marinas.

En el caso específico de los fondos marinos, FAO publicó en 2009 el documento "Directrices internacionales para la ordenación de las pesquerías de aguas profundas en alta mar". Este documento viene a colación de la necesidad de aplicar la Resolución N N 61/105 de la Asamblea General de las Naciones Unidas (AGNU). Esta medida instaba a los países a evaluar si las actividades que realizaban sus buques en los fondos marinos en aguas internacionales tenían efecto negativo sobre los Ecosistemas Marinos Vulnerables (EMV's) y también fue la base para la Medida de conservación 22-06 de CCRVMA, aplicable en la zona de influencia del Tratado Antártico. Específicamente, exhorta a los Estados a que adopten inmediatamente medidas precautorias para la protección de los EMV (como los arrecifes de aguas frías) ante actividades pesqueras destructivas. Si bien correspondía que estas directrices fueran aplicadas a fondos marinos por fuera de las plataformas continentales, la presencia de este tipo de ecosistemas ya fue registrada en fondos correspondientes a aguas internacionales (Portela et al. 2012) pero que actualmente quedarían incluidos dentro de los nuevos límites exteriores de la PCA.

\section{DISCUSIÓN}

Una primera mirada sobre la legislación nacional existente indica un aumento en cantidad y calidad de instrumentos de manejo específicos para los fondos marinos en los últimos años, ya que anteriormente la legislación hacía referencia de manera indirecta a este ítem. Estas referencias involucraban conceptos generales tales como conservación de recursos, prevención de efectos dañosos sobre el entorno, gestión sostenible del ambiente, etc. Por ejemplo, este estudio menciona que en 1988 el CO.CI.TE.MA indicaba la necesidad de incrementar el conocimiento de la plataforma submarina mientras que la Ley sobre Espacios Marítimos de 1991 hace referencia a los fondos marinos a partir de la determinación de la PCA. Sin embargo, es con la Ley de creación de las AMP's Namuncurá-Banco Burdwood I y II y Yaganes (y con su ley asociada del Sistema Nacional de Áreas Marinas Protegidas) que se toman medidas que hacen un señalamiento directo sobre el ambiente bentónico. En ese sentido, hay que destacar que a pesar del beneficio per se que parece tener la implementación de las AMP's como instrumentos de conservación, dado el alto costo que implica el manejo adecuado de las mismas y el conflicto que pueden generar frente a 
otras actividades económicas, es necesario que se evalúen indicadores de su eficacia a lo largo del tiempo a fin de verificar el cumplimiento de los objetivos planteados (Hockings et al. 2006; Radovich 2019a). Igualmente, más allá del avance que significa la designación de las autoridades de aplicación para las distintas leyes mencionadas, la superposición de autoridades y agencias gubernamentales con competencias relacionadas en la temática, sumado en algunos casos a la falta de reglamentación en cuestiones operativas y financiamiento, ponen hoy un límite para su ejecución efectiva (Sala 2018; Foro para la Conservación del Mar Patagónico y Áreas de Influencia 2019; Radovich 2019b).

Asimismo, en la Argentina existen otras medidas que no son tomadas directamente en función de la protección del medio bentónico pero pueden ser consideradas como elementos que contribuyen a la protección de los fondos marinos. Por ejemplo, las zonas de vedas que restringen los arrastres de fondo pueden ser consideradas en términos pesqueros como similares a las AMP's (Fisher y Frank 2002; Alemany et al. 2013). Si bien el objeto de estas zonas no está directamente enfocado en la protección de los fondos marinos y los invertebrados que allí habitan, la prohibición de arrastres de fondo puede redundar en una protección indirecta de los mismos (Murawski et al. 2000; Grizzle et al. 2009; O’Boyle 2011). En ese sentido, en la PCA existen distintas zonas de veda dirigidas a la protección de distintos recursos pesqueros (Allega et al. 2020) que en forma permanente o temporal pueden mitigar los efectos resultantes de la actividad pesquera sobre los fondos marinos.

Por otra parte, los planes de investigación y fomento nacionales diseñados desde 2010 en adelante involucran a los ambientes bentónicos como parte de áreas pasibles de explotaciones productivas (hidrocarburíferas o pesqueras) con un enfoque sostenible, y por ende reconocen la necesidad de profundizar en la investigación de dichos sectores. Esto se acopla a las distintas recomendacio- nes y resoluciones no vinculantes de organismos internacionales como FAO, que hacen hincapié en un enfoque ecosistémico en la explotación pesquera y por ello la necesidad de proteger especies y ambientes asociados. La cuestión radica en que esto implica un conjunto de herramientas dentro de las cuales el financiamiento y la formación de recursos humanos adecuados son primordiales. La sanción de la Ley PROMAR es un avance en ese sentido, ya que esta ley crea en su artículo 6 el Fondo Nacional para la Investigación e Innovación Productiva de los Espacios Marítimos Argentinos (FONIPROMA) con un piso anual presupuestario de 250 millones de pesos. Sin embargo, desde su sanción no se ha hecho una implementación efectiva de la partida presupuestaria asignada (Sala 2018).

Respecto a los tratados internacionales firmados con anterioridad (del Río de la Plata, CONVEMAR y CCRVMA), estos se refieren de forma directa a la exploración y explotación de recursos del lecho y subsuelo y a la protección del medio marino. Incluso en el caso de CCRVMA, implementan disposiciones específicas a través de medidas de conservación para la protección del ambiente bentónico. Debe reconocerse que todos los tratados fueron ratificados por leyes del Congreso Nacional, pero el amplio espectro de temas abarcados en dichas leyes produce que estas referencias queden desdibujadas, lo cual podría resolverse con una instrumentación más específica sobre la temática. Sin embargo, es un punto a favor que aunque las disposiciones de CCRVMA no sean aplicables en la PCA por no ser su ámbito de ejecución, sus fundamentos (ejemplo, prohibición de arrastres de fondo en zonas de existencia de EMV's) estén siendo utilizados en la justificación técnica para la creación de AMP's (DNC/APN 2017).

En relación a la ampliación de los límites exteriores de la plataforma continental, es necesario que nuestro país realice estudios sobre las particulares comunidades bentónicas que existen en parte del nuevo sector reconocido. Como se mencionó 
antes, en sectores que antes eran considerados fondos de alta mar, se registraron importantes arrecifes de corales de aguas frías que constituyen EMV's (Muñoz et al. 2012; Portela et al. 2012). Estas comunidades son equivalentes a las halladas en el Banco Burdwood y adyacencias y son un importante objeto de conservación a nivel mundial (Schejter et al. 2020). Por otra parte, especies de interés comercial como el cangrejo rojo (Chaceon notialis) y la langosta de profundidad (Thymops birsteini) se encuentran en fase de pesca experimental en zonas con profundidades cercanas a los $1.000 \mathrm{~m}$ en el lateral argentino por fuera de la Zona Común de Pesca Argentino-Uruguaya (Mauna et al. 2017). Si bien esta pesquería opera con artes pasivas como son las trampas o nasas, se ha comprobado que su uso implica también la colecta de invertebrados bentónicos, aunque con un bajo impacto sobre esta fauna (Mauna et al. 2018). Sin perjuicio de esto, el Acta $N^{\circ} 17 / 2017$ del CFP refiriéndose a la explotación en esa zona señala la importancia de "fortalecer el conocimiento que se tiene de la biodiversidad presente en los fondos marinos", lo cual habilita el estudio de estos organismos a fin de poder caracterizar las comunidades bentónicas presentes en el sector.

También, en virtud de la futura explotación hidrocarburífera en estas mismas zonas incluidas en los nuevos límites exteriores de la PCA, la Ley General del Ambiente indica que cualquier actividad pasible de dañar el medioambiente debe contar con evaluaciones de impacto ambiental previas a la realización de la mismas, así como además se exige la realización de audiencias públicas obligatorias. Estas prerrogativas son reconocidas en la Resolución Conjunta No 3/2019 firmada por la Secretaría de Energía y la Secretaría de Ambiente y Desarrollo Sustentable, donde además se cita la intervención del INIDEP como organismos técnico afectado para la revisión de los estudios de impacto ambiental. Por lo tanto, esto remarca que para cumplimentar eficientemente con las recomendaciones y reglamentaciones ambientales vigentes tanto en materia pesque- ra como de extracción de hidrocarburos es necesario realizar estudios enfocados en los invertebrados bentónicos que habitan en los nuevos límites exteriores de la PCA. Cabe señalar que estudios de este tipo ya han sido realizados por otros países en este sector (Muñoz et al. 2012; Portela et al. 2012). En ese sentido, es pertinente lo señalado por González (2012) sobre que "de poco sirve que el Estado $\mathrm{X}$ proclame su soberanía sobre recursos o su jurisdicción sobre actividades dentro de un dado número de millas marinas desde sus costas cuando instrumentos de dimensiones cada vez más reducidas, colocados por instituciones de la nacionalidad del Estado Y, que ingresan con creciente frecuencia en las aguas jurisdiccionales y hasta territoriales del Estado X, son los que permiten conocer la verdadera naturaleza de esos recursos o de esas actividades".

En definitiva, se puede afirmar que en los últimos años la Argentina ha avanzado en la generación de políticas de protección para los fondos marinos y especies asociadas, acorde a los lineamientos mundiales que implican un manejo ecosistémico de los recursos y un mayor resguardo de la biodiversidad (Tabla 3). Esto ha sido acompañado con el enunciado de distintos planes de investigación y fomento que involucran el estudio del ambiente marino y que incluyen a las comunidades bentónicas. Queda todavía pendiente en algunos casos la ejecución plena de las medidas debido a la superposición de facultades entre autoridades de aplicación, la falta de financiación y de plataformas de investigación acordes (buques científicos). También puede señalarse como pendiente la ausencia de planes de acción concretos referidos al estudio del lecho marino incluido en los nuevos límites exteriores de la PCA (sector por fuera de los $200 \mathrm{~m}$ de profundidad) donde, por el momento, solo se han realizado estudios aislados. Estos estudios pueden tener implicancias para la explotación sostenible de recursos vivos como también para el desarrollo de otras actividades productivas que puedan afectar los ecosistemas bentónicos. 
Tabla 3. Resumen de las distintas medidas enfocadas en todo o en parte a la protección directa o indirecta de los fondos marinos y su estado actual de ejecución.

Table 3. Summary of different measures focused in whole or in part on the direct or indirect protection of the seabed and their current state of implementation.

\begin{tabular}{llll}
\hline Medida & Tipo & Alcance & Estado de ejecución
\end{tabular}

Ley sobre Espacios
Marítimos No 23.968
(1991)

Constitución Nacional

(Art. 41) (1994)

Ley Federal de Pesca N ${ }^{\circ} \quad$ Ley

24.922 (1997)

Ley General del Ambiente Ley

$\mathrm{N}^{\circ} 25.675$ (2002)

Ley Creación del Área

Marina Protegida

Namuncurá-Banco

Burdwood No 26.875

(2013)

Ley Sistema Nacional de

Áreas Marinas

Protegidas $N^{\circ} 27.037$

(2014)

Ley PROMAR No 27.167

(2015)

Ley Creación de Áreas

Marinas Protegidas

Banco Burdwood II y

Yaganes No 27.490

(2018)

Área de veda pesca Banco Burdwood $^{1}$

Área de veda permanente merluza común (efectivo sur) ${ }^{1}$

Área de veda especies demersales costeras "El Rincón"1

Área de veda para pesca con arrastre de fondo en $\mathrm{ZCP}^{1}$

Ley

Ley

Ley

Ley

Ley

Ley
Nacional

Nacional

Nacional

En vigencia

Nacional

Nacional

Nacional

Disposición SSPyA
No $^{\circ} 250 / 2008$

Resolución CFP N ${ }^{\circ}$ 26/2009

Resolución CFP
27/2009
Resolución
CTMFM No
7/1997

\section{En vigencia}

Nacional

En vigencia

Nacional

En vigencia

Nacional En vigencia

Binacional En vigencia
En vigencia. Se designó como Autoridad de aplicación a la Secretaria de Agricultura, Ganadería, Pesca y Alimentación (actualmente Subsecretaría de Pesca y Acuicultura) (Decreto No 248/1998). Se reglamentó por Decreto $\mathrm{N}^{\circ} 748 / 99$.

En vigencia. Se designó como Autoridad de aplicación a la Secretaría de Ambiente y Desarrollo Sustentable (Decreto No 481/2003).

En vigencia. Se designó como Autoridad de aplicación a la Jefatura de Gabinete de Ministros (Decreto No 720/2014). Cambió su estatus, incluida actualmente en el Sistema Nacional de Áreas Marinas Protegidas (Decreto No 888/2019).

En vigencia. Se designó como Autoridad de aplicación a la Administración de Parques Nacionales (APN) (Decreto No 402/17).

En vigencia. Se designó como Autoridad de aplicación al Ministerio de Ciencia, Tecnología e Innovación Productiva (Decreto No 604/2016).

En vigencia. Incluidas actualmente en el Sistema Nacional de Áreas Marinas Protegidas. 
Tabla 3. Continuación.

Table 3. Continued.

\begin{tabular}{|c|c|c|c|}
\hline Medida & Tipo & Alcance & Estado de ejecución \\
\hline $\begin{array}{l}\text { Área de veda para la con- } \\
\text { servación de peces carti- } \\
\text { laginosos }\end{array}$ & $\begin{array}{l}\text { Resolución } \\
\text { CTMFM N } \\
8 / 2007\end{array}$ & Binacional & En vigencia \\
\hline $\begin{array}{l}\text { Área de veda estacional } \\
\text { merluza común (efectivo } \\
\text { norte) })^{1}\end{array}$ & $\begin{array}{l}\text { Resolución } \\
\text { CTMFM No } \\
1 / 1995\end{array}$ & Binacional & En vigencia \\
\hline $\begin{array}{l}\text { Tratado del Río de la } \\
\text { Plata (Ley N }{ }^{\circ} \\
20.645 / 1973)\end{array}$ & Tratado & Binacional & $\begin{array}{l}\text { En vigencia. La Comisión Técnica Mixta del Frente } \\
\text { Marítimo es la autoridad conformada para entender } \\
\text { en la temática (Art. } 80 \text { ). }\end{array}$ \\
\hline $\begin{array}{l}\text { Convenio Diversidad } \\
\text { Biológica (Ley N } \\
\text { 24.375/1994) }\end{array}$ & Tratado & $\begin{array}{l}\text { Internacional } \\
\text { (por países } \\
\text { firmantes) }\end{array}$ & $\begin{array}{l}\text { En vigencia. Se designó como Autoridad de } \\
\text { Aplicación a la Secretaría de Recursos Naturales y } \\
\text { Desarrollo Sustentable y se creó la Comisión } \\
\text { Nacional Asesora para la Conservación y } \\
\text { Utilización Sostenible de la Diversidad Biológica } \\
\left.\text { (Decreto } N^{\circ} 1347 / 97\right) \text {. Posteriormente se adoptó la } \\
\text { Estrategia nacional sobre diversidad biológica } \\
\text { (Resolución } N^{\circ} 91 / 2003 \text { ). }\end{array}$ \\
\hline $\begin{array}{l}\text { Convención de las } \\
\text { Naciones Unidas sobre } \\
\text { el Derecho del Mar } \\
\text { (CONVEMAR) (Ley No } \\
\text { 24543/1995) }\end{array}$ & Tratado & $\begin{array}{l}\text { Internacional } \\
\text { (por países } \\
\text { firmantes) }\end{array}$ & En vigencia \\
\hline $\begin{array}{l}\text { Comisión para la } \\
\text { Conservación de los } \\
\text { Recursos Vivos Marinos } \\
\text { Antárticos (CCRVMA) } \\
\text { (Ley No } 25.263 / 2000)\end{array}$ & Tratado & $\begin{array}{l}\text { Internacional } \\
\text { (por países } \\
\text { firmantes) }\end{array}$ & En vigencia \\
\hline $\begin{array}{l}\text { Código de Conducta para } \\
\text { la Pesca Responsable } \\
\text { (1995) }\end{array}$ & $\begin{array}{l}\text { Recomendación no } \\
\text { vinculante FAO }\end{array}$ & $\begin{array}{l}\text { Internacional } \\
\text { (de } \\
\text { aplicación } \\
\text { voluntaria) }\end{array}$ & $\begin{array}{l}\text { En su marco de aplicación se ha elaborado el Plan de } \\
\text { Acción Nacional para Prevenir, Desalentar y } \\
\text { Eliminar la Pesca Ilegal, No Declarada y No } \\
\text { Reglamentada (PAN-INDNR) y los Planes de } \\
\text { Acción Nacionales (PAN) para Aves, Mamíferos y } \\
\text { Tiburones. Además se instruyó al INIDEP para que } \\
\text { sus programas de investigación se estructuren bajo } \\
\text { un enfoque ecosistémico (Acta CFP N }{ }^{\circ} \text { 26/2006). }\end{array}$ \\
\hline $\begin{array}{l}\text { Enfoque ecosistémico } \\
\text { para la pesca }(2003)\end{array}$ & $\begin{array}{l}\text { Recomendación no } \\
\text { vinculante FAO }\end{array}$ & $\begin{array}{l}\text { Internacional } \\
\text { (de } \\
\text { aplicación } \\
\text { voluntaria) }\end{array}$ & Ídem anterior \\
\hline $\begin{array}{l}\text { Directrices internacionales } \\
\text { para la ordenación de las } \\
\text { pesquerías de aguas pro- } \\
\text { fundas en alta mar } \\
(2009)\end{array}$ & $\begin{array}{l}\text { Recomendación no } \\
\text { vinculante FAO }\end{array}$ & $\begin{array}{l}\text { Internacional } \\
\text { (de } \\
\text { aplicación } \\
\text { voluntaria) }\end{array}$ & $\begin{array}{l}\text { En función de cumplir la Resolución } \mathrm{N}^{\circ} 61 / 105 \text { de la } \\
\text { Asamblea General de las Naciones Unidas (AGNU) } \\
\text { se elaboró esta recomendación que luego fue la } \\
\text { base para la Medida de conservación 22-06 de } \\
\text { CCRVMA aplicable en la zona de influencia del } \\
\text { Tratado Antártico, del cual la Argentina es parte. }\end{array}$ \\
\hline
\end{tabular}


Tabla 3. Continuación.

Table 3. Continued.

\begin{tabular}{lccc}
\hline Medida & Tipo & Alcance & Estado de ejecución \\
\hline Agenda 2030 de & Recomendación no & Internacional & En el marco del Objetivo 14 y con el fin de proteger \\
Objetivos de Desarrollo & vinculante & al menos el 10\% de las zonas marinas y costeras se \\
Sostenible (2015) & AGNU & aplicación & crearon tres AMP's en la PCA. \\
& (Resolución $\mathrm{N}^{\mathrm{o}}$ & voluntaria) & \\
& $70 / 1)$ & & \\
\hline
\end{tabular}

${ }^{1}$ Áreas con prohibición permanente o temporal para arrastres de fondo (solo se señalan las de mayor extensión y persistencia en el tiempo). Se menciona la medida original, para ver modificaciones y ampliaciones vigentes consultar Allega et al. 2020.

\section{AGRADECIMIENTOS}

A los revisores que con sus valiosos comentarios estimularon un mayor grado de análisis sobre la propuesta original. A la Dra. Victoria González Carman por su impulso inicial en la elaboración de esta revisión y al Dr. Armando Abruza por sus comentarios que clarificaron algunos de los conceptos expresados en este trabajo. Al INIDEP que permitió la presentación en forma preliminar en forma oral en el XVIII Congreso Latinoamericano de Ciencias del Mar 2019 en la ciudad de Mar del Plata, Argentina. Contribución INIDEP No 2220.

\section{REFERENCIAS}

Alemany D, Iribarne OO, Acha EM. 2013. Effects of a large-scale and offshore marine protected areas on the demersal fish assemblage in the Southwest Atlantic. ICES J Mar Sci. 70: 123-134.

Allega L, Braverman M, Cabreira AG, Campodónico S, Carozza CR, CEPEDA GD, ColoNello JH, Derisio C, Di Mauro R, Firpo CA, et al. 2020. Estado del conocimiento biológico pesquero de los principales recursos vivos y su ambiente, con relación a la exploración hidro- carburífera en la Zona Económica Exclusiva Argentina y adyacencias. Mar del Plata: Instituto Nacional de Investigación y Desarrollo Pesquero (INIDEP). 119 p.

Auster PJ, Malatesta RJ, Langton RW, Watting L, Valentine PC, Donaldson CLS, Langton EW, Shepard AN, BabB WG. 1996. The impacts of mobile fishing gear on seafloor habitats in the gulf of Maine (Northwest Atlantic): Implications for conservation of fish populations. Rev Fish Sci. 4 (2): 185-202. Consejo Federal Pesquero. 2017. Acta CFP N ${ }^{\circ}$ 17/2017. Ciudad Autónoma de Buenos Aires, Argentina. $17 \mathrm{p}$.

[CCRVMA] COMISIÓN PARA LA CONSERVACIÓN DE los Recursos Vivos Marinos Antárticos. 2009a. Medida de conservación 91-03. Hobart, Australia. 3 p.

[CCRVMA] COMISIÓN PARA LA CONSERVACIÓN DE los Recursos Vivos Marinos Antárticos. 2009b. Medida de conservación 22-06. Hobart, Australia. 8 p.

Collie JS, Hall SJ, Kaiser MJ, Poiner IR. 2000. A quantitative analysis of fishing impacts on shelf-sea benthos. J Anim Ecol. 69: 785-798.

CoOK R, Fariñas-Franco JM, Gell FR, Holt RHF, Holt T, Lindenbaum C, Porter JS, Seed R, Skates LR, Stringell TB, SanderSON WB. 2013. The substantial first impact of bottom fishing on rare biodiversity hotspots: a 
dilemma for evidence-based conservation. PLoS ONE 8 (8): e69904. doi:10.1371/journal. pone.0069904

[COPLA] Comisión Nacional del Límite ExteRior de la Plataforma Continental. 2017. El Límite más extenso de la Argentina y nuestra frontera con la humanidad. http://www. plataforma argentina.gov.ar/es/el$1 \% \mathrm{C} 3 \% \mathrm{ADmite}-\mathrm{m} \% \mathrm{C} 3 \% \mathrm{~A} 1 \mathrm{~s}$-extenso-de-laargentina-y-nuestra-frontera-con-lahumanidad.

Cryer M, Hartill B, O'shea S. 2002. Modification of marine benthos by trawling: toward a generalization for the deep ocean? Ecol Appl. 12: 1824-1839.

CuminetTi SI. 2020. Aspectos administrativos y legales de las áreas marinas, costeras e insulares administradas por la Estado Nacional a través de la Administración de Parques Nacionales. Rev Elect Inst Invest Ambrosio L Gioja. 24: 90-121.

[DNC/APN] DiRección NACIONAL DE CONSERVACiÓN/AdMINISTRACiÓN DE PARQUES NACIONALES. 2017. Sistema Nacional de Áreas Marinas Protegidas (Ley N ${ }^{\circ}$ 27.037): Justificación técnica para la creación de seis áreas Marinas Protegidas. Documento Técnico de la Dirección Nacional de Conservación (APN/MAyDS), Ciudad Autónoma de Buenos Aires. 52 p.

FAO. 2012. Estado de las áreas marinas y costeras protegidas en América Latina. Ed. Aylem Hernández Avila. REDPARQUES Cuba. Santiago de Chile. 620 p.

FISHER JAD, Frank KT. 2002. Changes in finfish community structure associated with an offshore fishery closed area on the Scotian Shelf. Mar Ecol Prog Ser. 240: 249-265.

Foro PARA LA CONSERVACIÓN DEl MAR PATAGÓNICO Y Áreas De Influencia. 2019. Taller Técnico sobre Implementación de Áreas Marinas Protegidas Nacionales en el Mar Argentino. Reporte final. Informe inédito. Buenos Aires. $78 \mathrm{p}$.

GonZÁlez A. 2012. (Re) encontrar el Mar:
Argentina y el conocimiento científico de los océanos. Buenos Aires: Catálogos. 252 p.

Grizzle R, Ward L, Mayer L, Malik M, CoOper A, Abeels H, Greene J, Brodeur M, RosenBerg A. 2009. Effects of a large fishing closure on benthic communities in the western Gulf of Maine: recovery from the effects of gillnets and otter trawls. Fish Bull. 6: 308-317.

Hockings M, Stolton S, Leverington F, DudLEY N, Courrau J. 2006. Evaluating effectiveness: a framework for assessing management effectiveness of protected areas. Gland and Cambridge: IUCN. 105 p.

JOHNSON KA. 2002. A review of national and international literature on the effects of fishing on benthic habitats. NOAA Technical memorandum NMFS-F/SPO-57.

Mauna C, Firpo C, Flores N, Mango V. 2017. Pesca experimental del cangrejo rojo (Chaceon notialis) y la langosta de profundidad (Thymops birsteini), Área I, 2017. Inf Camp INIDEP No 22/2017. 12 p.

Mauna C, Flores N, Mango V, Lértora P, FiRPo C. 2018. Fauna acompañante de cangrejo rojo (Chaceon notialis) en el Área I, 2017. Inf Invest INIDEP No $\mathrm{N}^{\circ}$ 45/2018. 9 p.

Muntadas A, Demestre M, De Juan S, Frid CLJ. 2014. Trawling disturbance on benthic ecosystems and consequences on commercial species: a northwestern Mediterranean case study. En: LleOnart J, Maynou F, editores. The ecosystem approach to fisheries in the Mediterranean and Black Seas. Sci Mar. 78 (1): 53-65.

Muñoz A, Cristobo J, Rios P, Druet M, PoloNio V, Uchupi E, Acosta J, Atlantis Group. 2012. Sediment drifts and cold-water coral reefs in the Patagonian upper and middle continental slope. Mar Petrol Geol. 36: 70-82.

Murawski SA, Brown R, LAI HL, Rago PJ. HENDRICKSON L. 2000. Large-scale closed areas as a fishery-management tool in temperate marine systems: the Georges Bank experience. Bull Mar Sci. 66 (3): 775-798. 
O’Boyle R. 2011. Benefits of marine protected areas and fisheries closures in the Northwest Atlantic. Can Tech Rep Fish Aquat Sci. 2948. $68 \mathrm{p}$.

Pampa Azul. 2017. Horizontes estratégicos para el Mar Argentino. Buenos Aires. 96 p.

Portela J, Acosta J, Cristobo J, Muñoz A, Parra S, Ibarrola T, Del Río JL, Vilela R, Ríos P, Blanco R. et al. 2012. Management strategies to limit the impact of bottom trawling on VMEs in the high seas of the SW Atlantic. En: Cruzado A, editor. Marine Ecosystems. In Tech. p. 199-228.

RADOVICH V. 2018. Innovación en las estructuras de gobernanza hacia la gestión integral del mar en la República Argentina: consideraciones sobre las áreas marinas protegidas. Rev Elect Inst Invest Ambrosio L Gioja. 20: 223-247.

RADOVICH V. 2019a. Indicadores de medición de eficacia de las áreas marinas protegidas (AMPs). Obs Medioambient. 22: 75-90.

Radovich V. 2019b. Gestión ambiental del Mar en la República Argentina: reflexiones sobre los parques interjurisdiccionales marino costeros y las áreas marinas protegidas. Gest Amb. 22 (1). doi:10.15446/ga.v22n1.77181

Rossi S, Bramanti L, Gori A, Orejas C. 2017.
Animal forests of the world: an overview. En: Rossi S, Bramanti L, Gori A, OREJAs C, editores. Marine animal forests. The ecology of benthic biodiversity hotspots. Springer. 28 p. doi:10.1007/978-3-319-21012-4_1

SAla JE. 2018. Pampa Azul: el mar como territorio. Cienc Tecnol Polít. 1: 31-38.

Schejter L, Genzano G, Gaitán E, Perez CD, BREMEC CS. 2020. Benthic communities in the Southwest Atlantic Ocean: Conservation value of animal forests at the Burdwood Bank slope. Aquatic Conserv Mar Freshw Ecosyst. 30: 426-439.

Thrush SF, DAYTON PK. 2002. Disturbance to marine benthic habitats by trawling and dredging: Implications for marine biodiversity. Annu Rev Ecol. 33: 449-473.

Thrush SF, Gray JS, Hewitt JE, Ugland KI. 2006. Predicting the effects of habitat homogenization on marine biodiversity. Ecol Appl. 16: 1636-1642. doi:10.1890/1051-0761(2006) 016[1636:PTEOHH]2.0.CO;2

Tillin HM, HiddinK JG, Jennings S, Kaiser MJ. 2006. Chronic bottom trawling alters the functional composition of benthic invertebrate communities on a sea-basin scale. Mar Ecol Prog Ser. 318: 31-45. 
